\title{
Optimierung eines neuentwickelten Sensorkopfes für ein Tian-Calvet-Kalorimeter
}

\author{
Franz Schubert ${ }^{1}$, Michael Gollner ${ }^{2}$, Jaroslaw Kita ${ }^{1}$, Florian Linseis², Ralf Moos ${ }^{1}$ \\ ${ }^{1}$ Lehrstuhl für Funktionsmaterialien, Universität Bayreuth, 95447 Bayreuth, Deutschland \\ ${ }^{2}$ LINSEIS Messgeräte GmbH, 95100 Selb, Deutschland
}

\section{Zusammenfassung}

Dieser Beitrag zeigt die Optimierung eines Wärmestromsensors für ein Tian-Calvet-Kalorimeter. Im Gegensatz zur etablierten Technik wird der Sensor nicht aus geschweißten Drähten, sondern mittels der keramischen Mehrlagentechnik hergestellt. Der Sensor besteht aus einzelnen scheibenförmigen Sensorelementen, die abwechselnd mit porösen Alumosilikatisolationsringen gestapelt und in Serie verschaltet werden. Nachdem in Vorarbeiten gezeigt werden konnte, dass es sich um einen vielversprechenden Ansatz handelt, wurde die Empfindlichkeit des Sensors mittels der FiniteElemente-Methode optimiert. Zur Verifizierung des Modells wurden Messungen verschiedener Varianten des Sensors in Abhängigkeit der Temperatur durchgeführt und anschließend eine neue Gestalt der Sensorscheibe entworfen und getestet. Es konnte eine Steigerung der Empfindlichkeit um den Faktor 3,6 auf $29,5 \mu \mathrm{V} / \mathrm{mW}$ und die Verbesserung der Auflösung um den Faktor 10 auf $5 \times 10^{-7} \mathrm{~W}$ bei einer Zeitkonstante von $42 \mathrm{~s}$ erreicht werden. Der Sensor befindet sich somit auf dem Niveau kommerzieller Geräte.

Keywords: Kalorimetrie, Calvet, LTCC, Mehrlagentechnik

\section{Einleitung}

Kalorimeter sind in vielen Gebieten der Materialwissenschaft und der chemischen Reaktionstechnik weit verbreitete Messgeräte. Sie werden dazu genutzt, Wärmemengen von chemischen oder physikalischen Vorgängen zu quantifizieren. Mit inrer Hilfe kann der Verlauf von chemischen Reaktionen, die Wärmekapazität verschiedenster Materialien oder deren Schmelzenthalpie bestimmt werden [1]. Eine spezielle Variante solcher Geräte ist die Bauform nach Tian und Calvet [2]. Ihre Besonderheit liegt in der großen Anzahl drahtförmiger Thermoelemente (mehrere hundert), welche nach dem ThermosäulenPrinzip zu zwei turmförmigen Sensoren verschaltet werden. Der empfindliche Bereich der Sensoren hat einen Durchmesser von etwa $20 \mathrm{~mm}$ und eine Höhe von etwa $100 \mathrm{~mm}$. Der Vorteil dieser Geräte besteht im großen Probenvolumen, bei gleichzeitig hoher volumenspezifischer Empfindlichkeit. Dies erlaubt die Verwendung einer Vielzahl von Versuchseinrichtungen, zu denen Küvetten mit internen Mischsystemen oder Hochdruckeinrichtungen für Adsorptionsmessungen zählen [3].

In der vorliegenden Arbeit soll die Empfindlichkeit solcher Geräte durch eine Verbesserung der Wärmestromsensoren gesteigert werden. In Vorarbeiten dazu wurde ein neuartiger Ansatz zur Herstellung der
Wärmestromsensoren vorgestellt, der auf der Verwendung der keramischen Mehrlagentechnik basiert $[4,5,6,7]$. Dazu wurde der Sensor aus einem Stapel von empfindlichen Scheiben (Abb. 1) aus Low Temperature Cofired Ceramics (LTCC) und porösen Abstands-haltern aus Alumosilikatsteinen aufgebaut. Auf den Sensorscheiben wurden jeweils 34 Gold/Platin-Thermoelemente aufgebracht und jeweils acht Scheiben zu einer Thermosäule in Reihe verschaltet. Bei ersten Tests wurden zwei Sensoren in einen thermostatisierten Block verbracht und mittels elektrisch beheizter Probenküvetten die Empfindlichkeit bestimmt.

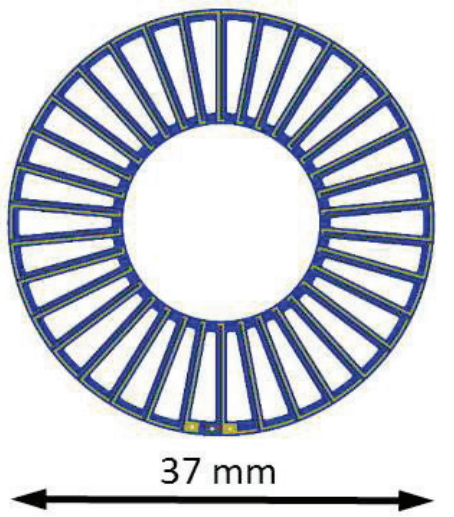

Abb. 1: Erste Generation der Sensorscheibe mit 34 Thermoelementen. 
Die Funktion des Aufbaus konnte in ersten Messungen bei Raumtemperatur nachgewiesen werden, wobei eine Empfindlichkeit von $8 \mu \mathrm{V} / \mathrm{mW}$ erreicht wurde.

\section{Methoden und Materialien}

Aufbauend auf dieser Arbeit wurde die Optimierung des Sensoraufbaus durchgeführt. Es wurden verschiedene Parameter beim Aufbau der Sensoren variiert zu denen Anzahl der Scheiben im Stack, Substratmaterial der Scheiben und Material der Isolation zählen. Zusätzlich wurde die Messtemperatur im Bereich zwischen Raumtemperatur und $400{ }^{\circ} \mathrm{C}$ variiert. Anschließend wurde ein FE-Modell des Stacks erstellt (Abb. 2), welches einen Ausschnitt mit dem dazugehörigen Substratmaterial und der Isolation beschreibt. Nach Verifizierung mit den vorhandenen Messdaten wurde das Modell dazu genutzt, eine neue, optimierte Sensorscheibe zu entwickeln.

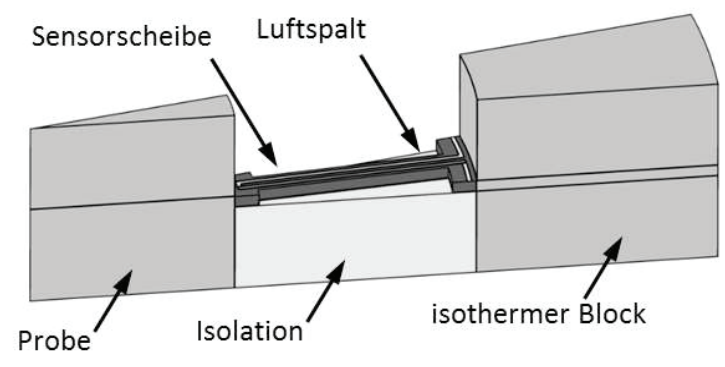

Abb. 2: Aufbau des Modells für die Optimierung.

\section{Ergebnisse und Diskussion}

Abb. 3 zeigt den Vergleich zwischen Messungen und dem Modell. Die Werte für die Empfindlichkeit stimmen für jede Temperatur auf $10 \%$ überein. Das Modell kann somit für die Optimierung des Sensoraufbaus verwendet werden. Bei allen Scheibenanzahlen steigt die Empfindlichkeit bei einer Temperaturerhöhung von $20^{\circ} \mathrm{C}$ auf $400{ }^{\circ} \mathrm{C}$ um den Faktor $2 \pm 0,15$. Den größten Einfluss hat hierbei die Steigerung des Seebeck-Koeffizienten des Gold-Platin-Thermoelementes, welcher in diesem Temperaturbereich um den Faktor 2,25 ansteigt [8]. Die Empfindlichkeit steigt nicht linear mit der Anzahl der Scheiben im Sensor, sondern flacht mit zunehmender Scheibenanzahl ab. Dies liegt an der höheren Wärmeleitfähigkeit der LTCC-Keramik im Vergleich zum Isolationsmaterial. Sie bewirkt, dass sich mit zunehmender Anzahl der Scheiben die vom kalorischen Vorgang hervorgerufene Temperaturänderung verringert.

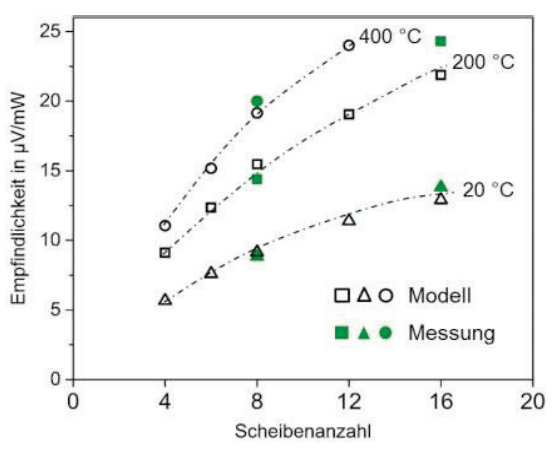

Abb. 3: Vergleich zwischen Messung und
Modell.

Es wurde eine neue Gestalt der Sensorscheibe entworfen, welche im Modell einen positiven Einfluss auf die Empfindlichkeit des Sensors hat. Die Empfindlichkeit steigt, wie bei der nicht optimierten Scheibe, mit der Anzahl der Sensorscheiben, wobei der Anstieg ebenfalls bei höheren Scheibenzahlen abflacht. Im Vergleich zur nicht optimierten Scheibe verbessert sich die Empfindlichkeit um den Faktor 2. Messungen an Sensoren mit acht optimierten Sensorscheiben zeigen deutlich verbesserte Eigenschaften gegenüber der ersten Generation. Die erste Generation der Sensoren mit 34 Thermoelementen zeigt dabei eine Empfindlichkeit von $8,3 \mu \mathrm{V} / \mathrm{mW}$ mit einer Detektionsgrenze von $5 \times 10^{-6} \mathrm{~W}$. Durch die neue, optimierte Sensorscheibe mit 67 Thermoelementen wurde die Empfindlichkeit auf $17,9 \mu \mathrm{V} / \mathrm{mW}$ angehoben. Die Detektionsgrenze verbesserte sich dabei zunächst jedoch nicht. Durch weitere Optimierungsmaßnahmen im Sensor konnte die Empfindlichkeit nochmals gesteigert werden. Ein noch weiter verbesserter Aufbau zeigte eine Empfindlichkeit von 29,5 $\mu \mathrm{V} / \mathrm{mW}$. Die Detektionsgrenze konnte dabei auf $5 \times 10^{-7}$ $W$ um eine Dekade verbessert werden.

Abb. 5 zeigt eine Messkurve der optimierten Sensorscheibe mit 67 Thermoelementen im optimierten Sensor bei Raumtemperatur. Das Signal wurde mit einem gleitenden Durchschnitt von 10 Werten geglättet, wobei das Rauschen des Ausgangssignals etwa $50 \mathrm{nV}$ rms beträgt und die Basislinie weniger als $\pm 50 \mathrm{nV}$ schwankt. Die Zeitkonstante des Sensors beträgt $T_{1 / \mathrm{e}}=42 \mathrm{~s}$. 


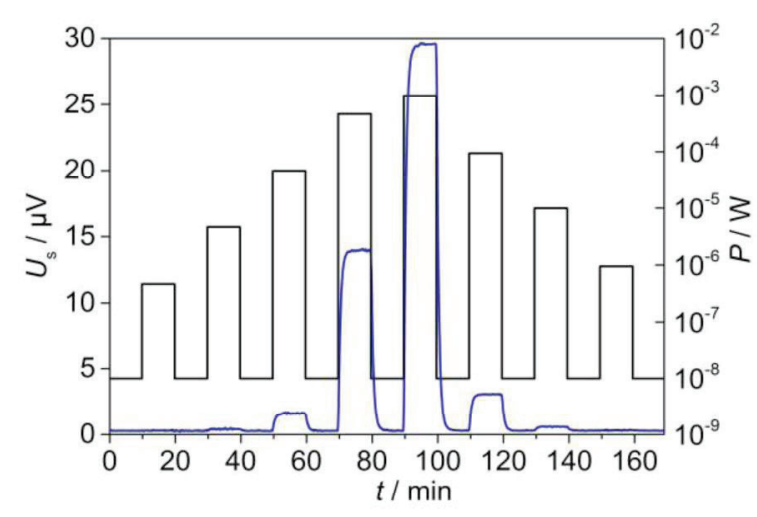

Abb. 5: Die Sensorspannung $U_{s}$ steigt mit der optimierten Scheibe deutlich an.

\section{Zusammenfassung und Ausblick}

Im Beitrag wurde eine Optimierung eines Wärmestrom-sensors für ein Tian-CalvetKalorimeter gezeigt. Zunächst wurde ein Modell mittels der Finite-Elemente-Methode erstellt und mit Messungen bestätigt. Die Abweichung zwischen Modell und Messungen beträgt weniger als $10 \%$, sodass das Modell zur Optimierung der Sensorscheibe und des Sensors geeignet ist. Durch Erhöhung der Anzahl von Thermoelementen in der Sensorscheibe konnte die Empfindlichkeit des Aufbaus von $8,3 \mu \mathrm{V} / \mathrm{mW}$ auf $17,9 \mu \mathrm{V} / \mathrm{mW}$ gesteigert werden. Zusätzlich ermöglichen weitere Verbesserungen am Sensor eine Steigerung der Empfindlichkeit auf $29,5 \mu \mathrm{V} / \mathrm{mW}$. Insgesamt wurde somit eine Steigerung der Empfindlichkeit um den Faktor 3,6 erreicht. Die Auflösung wird mittels der neuen Gestalt des Sensors um den Faktor 10 auf $5 \times 10^{-7} \mathrm{~W}$ verbessert. Die Zeitkonstante $T_{1 / \mathrm{e}}=42 \mathrm{~s}$ liegt deutlich unterhalb derer von kommerziellen Geräten, welche Werte von $280 \mathrm{~s}$ bis $360 \mathrm{~s}$ aufweisen [9]. Der Messaufbau zeigt damit eine vergleichbare Performance wie kommerzielle Geräte, welche eine Empfindlichkeit von $30 \mu \mathrm{V} / \mathrm{mW}$ und eine Auflösung von $0,1 \mu \mathrm{W}$ aufweisen [10]. Im Weiteren soll die Empfindlichkeit durch Verwendung neuer Thermopaare gesteigert werden und der Prototyp eines Laborgerätes aufgebaut werden.

\section{Literatur}

[1] G. Höhne, W. Hemminger, H.-J. Flammersheim, Differential scanning calorimetry: An introduction for practitioners, Springer, Berlin, New York (2003)

[2] É. Calvet, H. Prat, Microcalorimétrie: Applications physico-chimiques et biologiques, Masson (1956)

[3] A. Auroux (Ed.), Calorimetry and thermal methods in catalysis, Springer-Verlag, Berlin Heidelberg (2013)
[4] M. Gongora-Rubio, P. Espinoza-Vallejos, L. Sola-Laguna, J. Santiago-Avilés, Overview of low temperature co-fired ceramics tape technology for meso-system technology (MsST), Sensors and Actuators A: Physical 89, 222-241 (2001); doi:10.1016/S09244247(00)00554-9

[5] F. Schubert, M. Gollner, J. Kita, F. Linseis, R. Moos, First steps to develop a sensor for a Tian-Calvet calorimeter with increased sensitivity, JSSS (2016), in Review

[6] J. Kita, R. Moos, Development of LTCCmaterials and their applications - an overview, Informacije MIDEM 38, 219-224 (2008)

[7] J. Kita, F. Rettig, R. Moos, K.-H. Drue, H. Thust, Hot Plate Gas Sensors-Are Ceramics Better?, Int J Applied Ceramic Technology 2, 383-389 (2005); doi:10.1111/j.1744-7402.2005.02037.x

[8] M. Gotoh, K.D. Hill, E.G. Murdock, A gold/platinum thermocouple reference table, Rev. Sci. Instrum. 62, 2778 (1991), doi:10.1063/1.1142213

[9] S. Barale, L. Vincent, G. Sauder, N. Sbirrazzuoli, Deconvolution of calorimeter response from electrical signals for extracting kinetic data, Thermochimica Acta 615, 30-37 (2015); doi:10.1016/j.tca.2015.07.007

[10] SETARAM Instrumentation, C80 specifications, $h$ ttp://www. setaram.com/C80Specifications.htm, zuletzt besucht: 10. Februar 2016

\section{Danksagung}

Die vorgestellten Ergebnisse wurden im Rahmen des Projektes „Hochauflösender, kalorimetrischer Sensor" im FuE-Programm Mikrosystemtechnik Bayern des Bayerischen Staatsministeriums für Wirtschaft und Medien, Energie und Technologie unter dem Förderkennzeichen MST-1304-0003 // BAY182/002 erzielt. 